\title{
Image of the month: An unusual cause of cough, stridor and dyspnoea: A giant aortic arch aneurysm
}

\author{
Authors: Bhupinder Singh, ${ }^{\mathrm{A}}$ Abhishek Goyal, ${ }^{\mathrm{A}}$ Shibba T Chhabra, ${ }^{\mathrm{B}}$ Naved Aslam, ${ }^{\mathrm{B}}$ Bishav Mohan ${ }^{\mathrm{B}}$ and \\ Gurpreet S Wander ${ }^{\mathrm{B}}$
}

\begin{abstract}
A 78-year-old man with hypertension presented with dry cough and gradually progressive dyspnoea for 3 months. The patient had an audible stridor. Cardiovascular examination was unremarkable. Respiratory system examination revealed both inspiratory and expiratory grunting sound. Laboratory investigations were normal. Electrocardiography showed sinus rhythm with left ventricular hypertrophy (LVH). Chest $\mathrm{X}$-ray showed superior mediastinal widening. Transthoracic echocardiography showed preserved LV functions and dilated aortic arch. Contrast-enhanced computed tomography of the thorax showed a huge aortic arch aneurysm compressing the adjacent trachea. The patient was planned for hybrid aortic arch repair but the patient refused and was discharged on antihypertensive, antiplatelet and statin therapy. The patient continued to have limiting cough, dyspnoea and stridor for 4 months of follow-up until he suddenly died while at home. This case highlights an unusual presentation of a potentially lethal disease. Evaluation of patients presenting with cough and dyspnoea should not be restricted to respiratory diseases. The critical observations made from history
\end{abstract}

KEYWORDS: dyspnoea, stridor, aortic aneurysm

DOI: $10.7861 /$ clinmed.2021-0095

\section{Case presentation}

A 78-year-old man with hypertension presented to the cardiology clinic with dry cough and gradually progressive dyspnoea for the previous 3 months. The patient also gave a history of grunting sound with respiration. He had no fever, chest pain, orthopnoea or pedal oedema. On examination, he had a pulse rate of 78 beats per minute, blood pressure of $168 / 82 \mathrm{mmHg}$ and normal peripheral arterial oxygen saturation. The patient had an audible stridor. Cardiovascular examination was unremarkable. Respiratory system examination revealed both inspiratory and expiratory grunting sound.

Routine laboratory investigations (including brain natriuretic peptide and cardiac enzymes) were normal. Electrocardiography

Authors: Aassociate professor, Dayanand Medical College and Hospital, Ludhiana, India; ${ }^{B}$ professor, Dayanand Medical College and Hospital, Ludhiana, India.

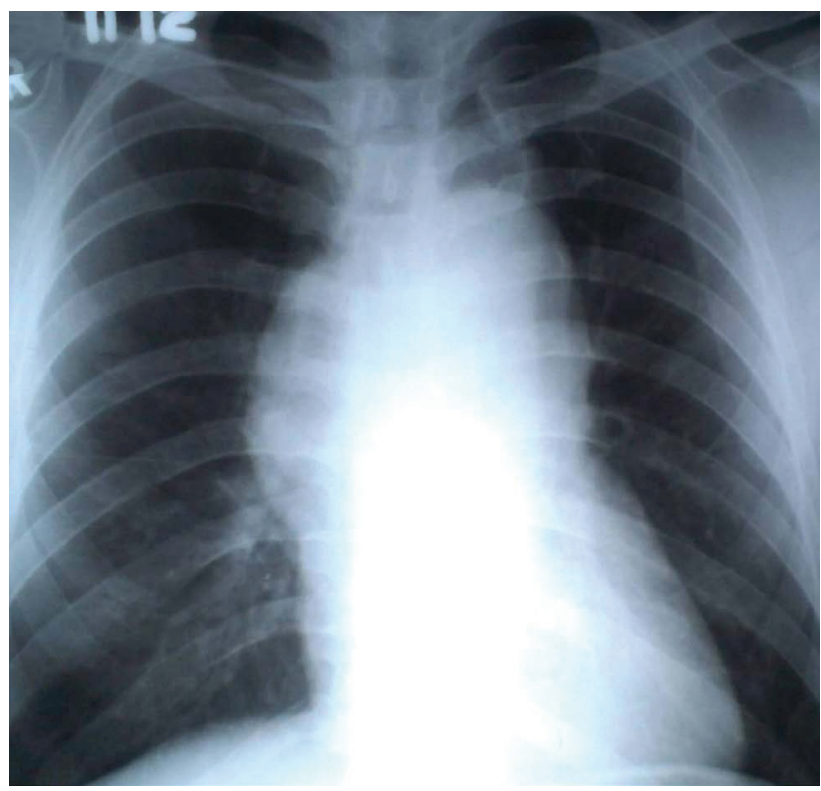

Fig 1. Chest X-ray showing superior mediastinal widening.

was sinus rhythm with left ventricular hypertrophy (LVH). Chest $X$-ray (Fig 1) showed superior mediastinal widening. Transthoracic echocardiography showed moderate concentric LVH, preserved LV functions and dilated aortic arch. Contrast-enhanced computed tomography (Fig 2a) of the thorax showed a huge aortic aneurysm $(10.9 \times 9.2 \mathrm{~cm})$ arising from the floor of the aortic arch, which was compressing the adjacent trachea (Fig 2b). The aneurysm was having thrombus at the periphery (Fig $2 b$ ). Coronary artery disease was ruled out on conventional coronary angiography. The patient was planned for hybrid aortic arch repair but he refused to give consent for it, therefore, he was discharged on antihypertensive, antiplatelet and statin therapy. The patient continued to have limiting cough, dyspnoea and stridor for 4 months of follow-up until he suddenly died while at home.

\section{Discussion}

With increasing life expectancy and better screening protocols, the incidence of thoracic aortic aneurysms (TAAs) is increasing. TAAs commonly involve ascending aorta $(60 \%)$ followed by descending 

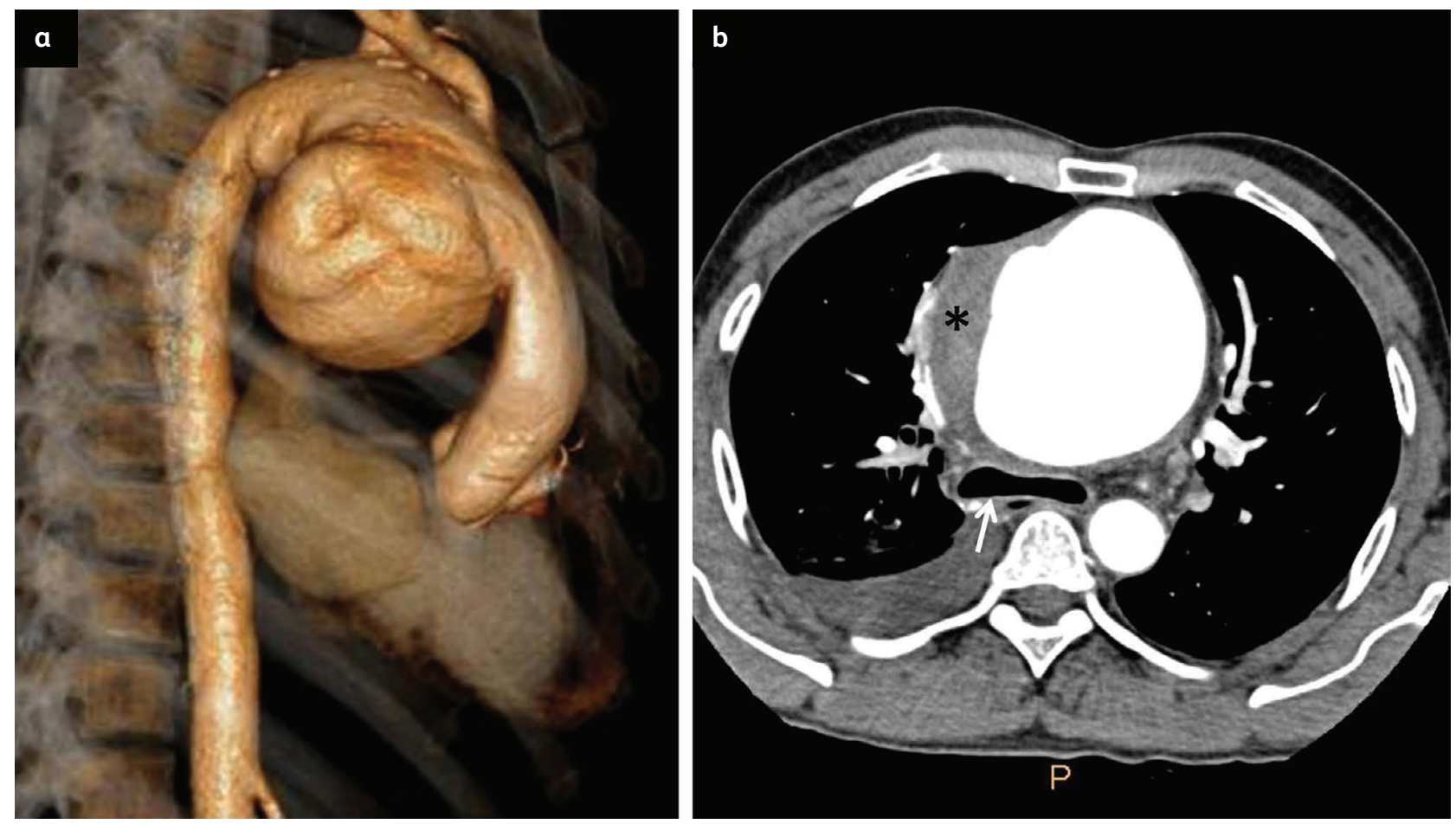

Fig 2. Contrast-enhanced computed tomography of the thorax. a) Computed tomography showing 3-dimensional reconstruction of an aortic arch aneurysm (measuring $10.9 \times 9.2 \mathrm{~cm}$ ). b) Computed tomography showing tracheal compression (white arrow) on its anterior aspect by the aortic arch aneurysm. Thrombus visible in the periphery of the aneurysm (black asterisk).

thoracic aorta $(40 \%)$, aortic arch $(10 \%)$ and thoracoabdominal aorta $(10 \%)$. Clinically most of the aortic aneurysms remain asymptomatic for a long time and are incidentally detected for imaging for other medical reasons. Sometimes giant thoracic aneurysms can have local mass effect; patients become symptomatic due to compression of adjacent structures such as vertebral bone erosion (back pain), oesophageal (causing dysphagia), recurrent laryngeal nerve (Ortner's syndrome), trachea or main bronchus (cough, dyspnea and stridor). Tracheo-bronchial compression by huge aortic arch aneurysm is mentioned in literature., ${ }^{1,2}$ Management of aortic arch aneurysms is challenging. ${ }^{3}$ Its repair is associated with higher morbidity and mortality rates compared with the treatment of ascending aortic aneurysms, owing to a high risk of cerebral complications. Nowadays hybrid aortic arch repair is preferred over only surgical repair.

\section{References}

1 MacGillivray RG. Tracheal compression caused by aneurysms of the aortic arch. Implications for the anaesthetist. Anaesthesia 1985;40:270-7.

2 Lin F, Chen CC, Su Y], Lai YC, Chang WH. Ruptured aortic aneurysm presenting as a stridor. Int J Gerontol 2010;4:96-8.

3 Crawford ES, Saleh SA, Schuessler JS. Treatment of aneurysm of transverse aortic arch. J Thorac Cardiovasc Surg 1979;78:383-93.

Address for correspondence: Dr Bhupinder Singh, Department of Cardiology, Dayanand Medical College and Hospital,

Ludhiana 141001, Punjab, India.

Email:dr_bhupinders@yahoo.in 